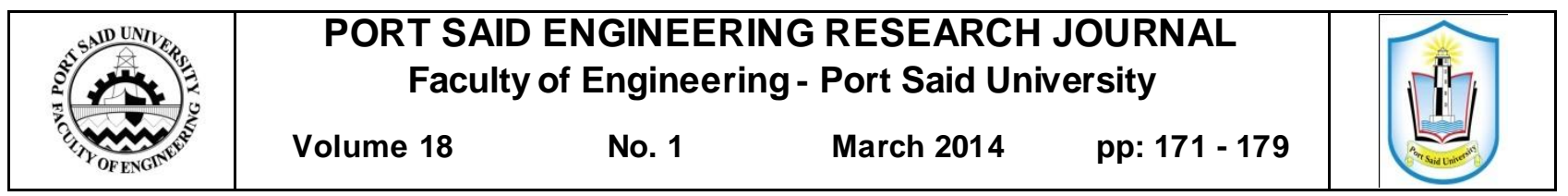

\title{
Improve Energy Efficiency through Nano Pore Vacuum Insulation Panels "Vips"
}

\section{Abstract}

\author{
Mustafa M. Abd El-Hafez ${ }^{1}$, AshrafEl-Mokadem ${ }^{2}$ and Noha M. Abu-Samra ${ }^{3}$
}

Energy efficiency of the built environment greatly depends on the performance of the insulating materials used in the building envelope construction. Vacuum insulation panels (VIPs) offer excellent thermal resistance properties that can enhance the energy efficiency of the insulating systems and provide savings in energy consumption. However, VIP systems are virtually unknown and rarely us ed for building construction. There is a need to investigate the use of VIPs in various components of building envelopes (walls - roofs) and their long-term function and performance.

\section{ENERGY EFFICIENCY}

Energy Efficiency is the capacity to produce results with a minimum overall expenditure of energy, human effort, materials and capital. Because the cost and availability of these resources differ, efficiency is relative to time and place. This means that countries have different perspective and limitations on efficiency, but share the same aim of minimizing expenditure of resources for the tasks at hand [1].

Energy efficiency is the capacity to produce results with a minimum expenditure of energy inputs. Waste energy is any energy in a system which is not directly serving the systems function. A system is thought of energy-efficient if its requirements are low in relation to the results produced, and if energy is not wasted [2].

\section{Energy Effiency - cheaper than new energy}

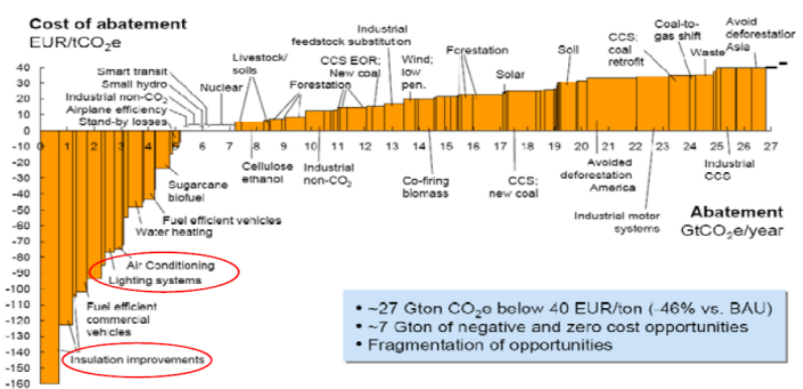

Figure (1): Energy efficiency cheaper than new energy (Cost for various GHG measures in 2030)

Source: Anne Grete Hestnes and Marit Thyholt, Zero Emission Buildings (ZEB), The Research Centre on Zero Emission Buildings, February 2009, www.zeb.no

\footnotetext{
${ }^{1}$ Architecture and Urban Planning Department, Faculty of Engineering, Port Said University, Port Said, Egypt, E-mail: whitehouse.egy51@yahoo.com

${ }^{2}$ Architecture and Urban Planning Department, Faculty of Engineering, Port Said University, Port Said, Egypt, E-mail: elmokadem1@gmail.com

3 Architecture and Urban Planning Department, Faculty of Engineering, Port Said University, Port Said, Egypt, E-mail: abosamranoha@yahoo.com
}

\section{VACUUM INSULATION PANELS (VIP)}

And thus, we can define energy efficiency as "the ability to provide the best results at the lowest possible energy sparingly, without prejudice to the job"

Minimu $\mathrm{m}$ Inputs Energy + Minimu $\mathrm{m}$ Waste Energy = Energy Efficiency

VIP are regarded as one of the most promising high performance thermal insulation solutions on the market today, Thermal performances five to ten times better than conventional insulation of the same thickness.

\subsection{Definition of VIPs}

A vacuum insulation panel is composed of two components: a micro- to nano- porous material called the "core" that is evacuated, and sealed using a thin membrane called the "foil" [3]. Vacuum insulation panels are defined as: "An evacuated foil-encapsulated open porous material as a high performance thermal insulating material" [4].

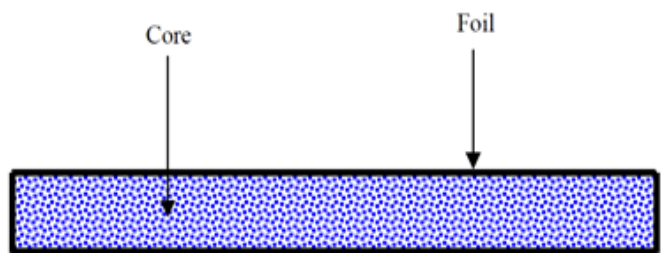

Figure (2): schematic representation of a vacuum insulation panel

Source: Mukhopadhyaya, P., High-performance insulation materials, National Research, Council Canada, September 2004.

\subsection{Components of VIPs}

VIP has three major components [5]:

- Core Material: imparts mechanical strength and thermal insulating capacity by preventing the free flow 
of the gas/air molecules thereby reducing the ability of heat transfer through air conduction, (e.g. fumed silica, aerogel, glass fiber or foams). Ideal core materials should have an open cell structure, very small pore diameter, resistance to compression due to atmospheric pressure and very high resistance to infrared radiation.

- Gas Barrier / Facer Foil: provides the air and vapour-tight enclosure for the core material. The longterm performance of the VIPs is very much dependent on the performance of the gas barrier of facer foil.

- Getter / Desiccant: is added inside the core material to adsorb residual or permeating atmospheric gases or water vapour in the VIP enclosure. The addition of getter/desiccant increases the performance and longevity of VIPs.

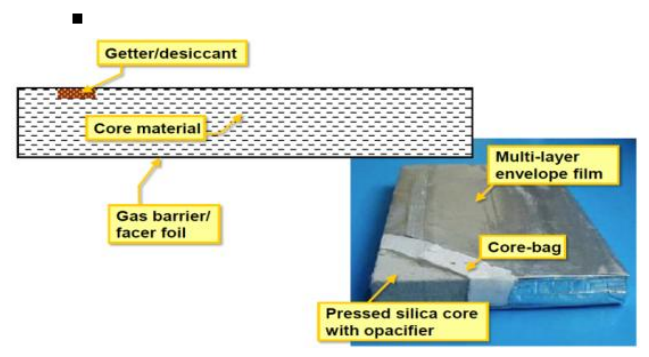

Figure (3): Components of "VIP"

Source: Dr. Phalguni Mukhopadhyaya, High-Performance Vacuum Insulation Panel in Building Envelope Construction, National Research, Council Canada, 2010 AMTS Technical Committee Meeting 06 October 2010. www.nrc-cnrc.gc.ca/irc

There are many various shapes possible of VIP, in particular for technical applications, which give vacuum insulation very good value.

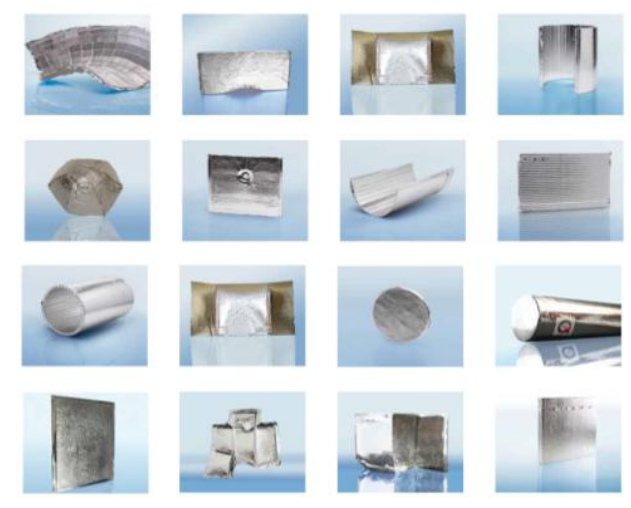

\section{Figure (4): Different shapes of vacuum insulation} panels

Source: Mukhopadhyaya, P., High-performance insulation materials, National Research, Council Canada, September 2004.

The thermal resistance of VIPs is several times (up to 10 times) higher than conventional insulating materials, therefore Thermal conductivity of VIPs is several times (up to 10 times) lower than conventional insulating materials.
Table 1: Thermal conductivity for different Insulator

\begin{tabular}{|c|c|}
\hline Insulation Methods & Ther mal conducti vity \\
\hline Traditional Insulation & $36 \mathrm{~mW} /(\mathrm{mK})$ \\
\hline Vacuum Insulation Panels & $4 \mathrm{~mW} /(\mathrm{mK})$ fresh \\
(VIP) & $8 \mathrm{~mW} /(\mathrm{mK}) 25$ years \\
& $20 \mathrm{~mW} /(\mathrm{mK})$ perforated \\
\hline Gas-Filled Panels (GFP) & $40 \mathrm{~mW} /(\mathrm{mK}$ \\
\hline Aerogels & $13 \mathrm{~mW} /(\mathrm{mK})$ \\
\hline
\end{tabular}

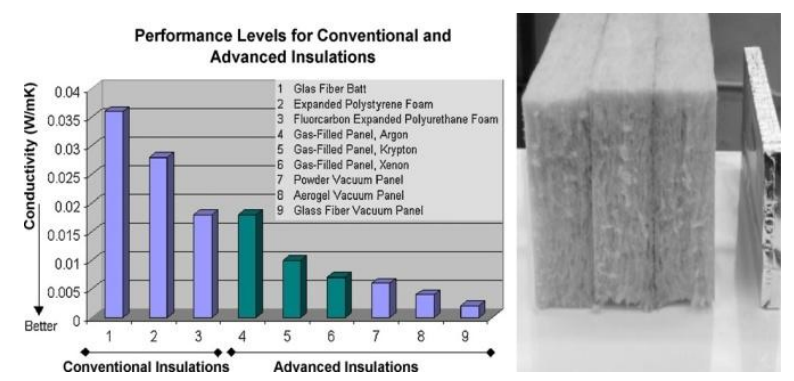

Figure (5): [left] the ther mal conducti vities of conventional and advanced insulation materials and

solutions and [right] the required thicknesses for

conventional insulation (e.g. glass wool) and a VIP Source: va-Q-tec c, Vacuum Insulation Panels (VIPs), June 2011, www.va-Q-tec.com

\subsection{Advantages of VIPs}

VIPs is considered the best solution today and in the near future. VIPs Advantages are:

- Higher thermal resistance.

- Reduced thickness of the component.

- Recyclable.

- High performance thermal insulating.

- Increased floor area.

- Appropriate refurbishing of existing buildings with high restrictions.

- Lower operating temperature increases thermal.

\subsection{Disadvantages of VIPs}

Although VIPs has many Advantages, there are many Disadvantages,

- Any damage in the vacuum system (even a small pinhole) will severely destroy the thermal insulating capacity of VIPs .

- Very fragile and protection for puncture of the foil is necessary.

- Reducing thermal performances through time.

- Limited service life may require replacement.

- Less suitable for traditional timber wall structures.

- Production inaccuracy of the panel sizes.

- Inflexible: Can not be cut or adapted at building site. 


\subsection{Challenges and solution of VIPs}

\begin{tabular}{|c|c|}
\hline Challenges & solution \\
\hline$\frac{\underline{\text { Cost }}}{\text { (re latively }}$ & $\begin{array}{l}\text { - Like many other nanomaterials cost } \\
\text { effective, high quality and large } \\
\text { volume production of nanoporous } \\
\text { insulating materials is important for } \\
\text { their widespread applications. }\end{array}$ \\
\hline 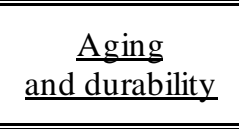 & $\begin{array}{l}\text { - Manufacturing } \\
\text { - Properties of core materials } \\
\text { - Handling and exposure } \\
\end{array}$ \\
\hline$\frac{\frac{\text { Thermal }}{\text { bridge effects at }}}{\underline{\text { edges }}}$ & $\begin{array}{l}\text { - Use large panels } \\
\text { - Overlap panels } \\
\text { - Fill gaps at edges with insulating } \\
\text { materials }\end{array}$ \\
\hline$\underline{\text { Condensation }}$ & $\begin{array}{l}\text { - VIP is an absolute vapour barrier } \\
\text { - Avoid damp construction materials } \\
\text { - Consequences of vacuum failure }\end{array}$ \\
\hline
\end{tabular}

\subsection{Lifespan of VIPs}

The life expectancy of a vacuum insulation panel is determined by a number of factors. Specifically, these are;

- The initial vacuum level of the panel.

- The permeation rate of the membrane film.

- The out-gassing (if any) of the core material and membrane film.

- The permeation rate of the membrane sealing edge

- The quantity and effectiveness of the getter and desiccant.

- The effect of pressure rise on the specific core material.

- Minimum Performance Requirements

- Panel Size

- Fabrication Quality

- VIP Component Choices

- Use Conditions.

The two primary factors that determine the long-term thermal performance of the vacuum insulation panel are:

- Effectiveness of gas barrier, and

- Performance of core material.

\subsection{Recommendations to Usages of VIPs}

To raise the confidence in VIP-technology and their use in building applications the following recommendations should be taken [6]:

- Industrial processing: integration of VIP in prefabricated build ing systems and components.

- Information and consulting: all concerned be informed, advised as early as possible and be supported by a specialist during the entire planning and installation process (preferably by the VIP supplier). Warning label "VIP inside".
- Handling: VIP must be handled with care and suitable protective measures and tools employed (protective mats, felt shoes, etc.).

- Detailing: VIP must be well protected from mechanical damage. This applies to functional loading (e.g. from the floor). Inadvertent loading (e.g. dilatation) and subsequent manipulations (e.g. nailing). One must pay special attention to various joint details, since protecting components (e.g. angle brackets for window attachment, guide rails, frames) may damage the VIP.

- Reducing the edge effect: select panels that are as square and large as possible $\left(\mathrm{min} .0 .5 \times 0.5 \mathrm{~m}^{2}\right)$. Panel with an aluminum foil envelope (only rarely nowadays), lay the panels in a double layer, overlapping by at least 5 $\mathrm{cm}$.

- Water vapour diffusion: VIP are vapour-tight insulation systems, which has to be taken into account in planning the order and thickness of the layers. Furthermore, special attention must be given to the joints between the panels.

- Inspection of the VIP: installation of the VIP in such a way that inspection of their correct functioning can be made (sensor or thermograph).

- Replace-ability: it would be desirable, that the VIP are embedded in the construction such that they can be replaced without undue effort in preparation or as a result (e.g. mechanically fixed covers). Otherwise deterioration in the U-value has to be accepted and it must be assured that on loss of vacuum, there is no risk of loss of condensation and mould at the inside of the walls.

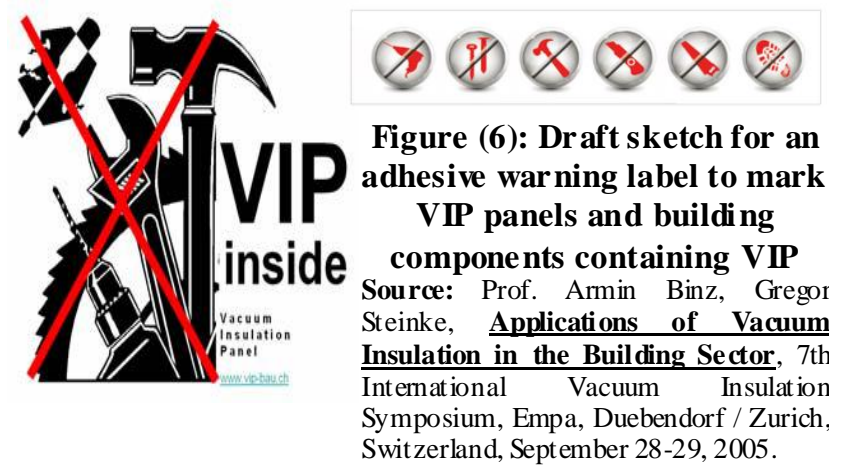

\subsection{Development of VIPs}

- Before 2005, VIP has only limited use, mainly in top models home refrigerators/freezers and cold shipping boxes. Japan controls more than $50 \%$ of the small global VIP market with several million panels per year. The VIP market in Japan is fast growing. The common core materials are fumed and precipitated silica. 


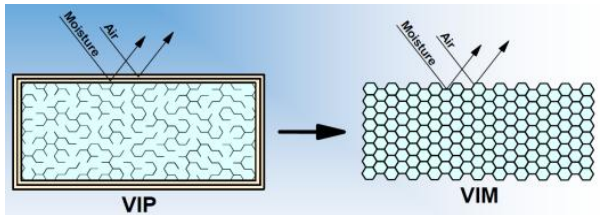

Figure (7): vacuum insulation panels before 2005 Source: Bjørn Petter Jelleab and Arild Gustavsenc, Advanced Thermal Building Insulation (From Vacuum Insulation Panels to Nano Insulation Materials), Trondheim, Norway, 9th of September, 2010.

- After 2005, Nano Insulation Material (NIM): A basically homogeneous material with a closed or open small nano pore structure with an overall thermal conductivity of less than $4 \mathrm{~mW} /(\mathrm{mK})$ in the pristine condition [4].
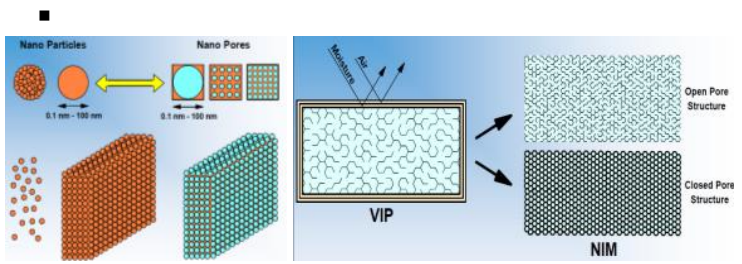

Figure (8): vacuum insulation panels after 2005 Source: Bjørn Petter Jelleab and Arild Gustavsenc, Advanced Thermal Building Insulation (From Vacuum Insulation Panels to Nano Insulation Materials), Trondheim, Norway, 9th of September, 2010.

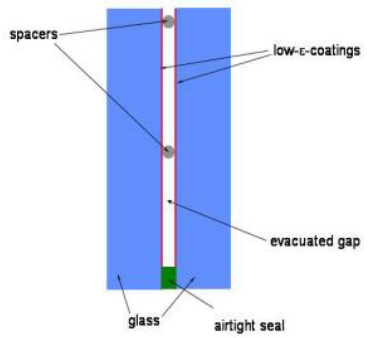

Figure (9): Princi ple of a Vacuum Insulation Glass Source: H. Weinlader, H.-P. Ebert, J. Fricke, VIG - Vacuum Insulation Glass, Bavarian Center for Applied Energy Research (ZAE Bayern), Am Hubland, D-97074 Wurzburg.

- In the near future, Vacuum Insulation Glass (VIG): VIG is a double glazing with a vacuum between the panels of glass. This means that slim systems less than $10 \mathrm{~mm}$ thick can be realized with heat transfer coefficients of $\mathrm{U} \approx 0.5 \mathrm{~W} /\left(\mathrm{m}^{2} \mathrm{~K}\right)$. Triple glazing, used, for instance, in passive houses, achieves a typical U-value of 0.6-0.7 $\mathrm{W} /\left(\mathrm{m}^{2} \mathrm{~K}\right)$ at a thickness of $28-44 \mathrm{~mm}$, but is $50 \%$ heavier. VIG with a $\mathrm{U}$-value of $\approx 0,5 \mathrm{~W} /\left(\mathrm{m}^{2} \mathrm{~K}\right)$ is not yet available on the market [7].

\section{IMPROVE ENERGY EFFICIENCY THROUGH NANO PORE VACUUM INSULATION PANELS "VIP"}

We can improve energy efficiency through opaque parts (wall sections) used nano insulation materials (nano pore vacuum insulation panels "VIP").

\subsection{Wall Sections Shape}

Wall Section Shape is divided into:

$\underline{\text { Solid Wall Section }(20 \mathrm{~mm}-120 \mathrm{~mm}-20 \mathrm{~mm})}$

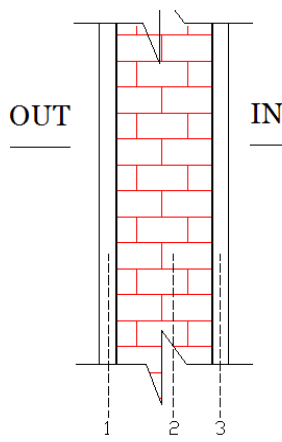

Without

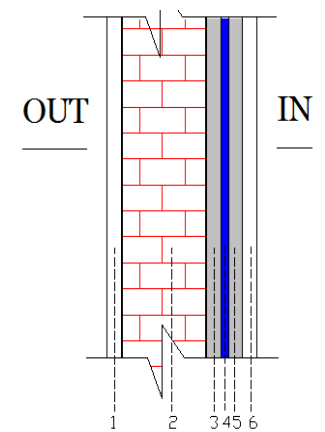

With (VIP) Insulation

$\underline{\text { Solid Wall Section }(20 \mathrm{~mm}-250 \mathrm{~mm}-20 \mathrm{~mm})}$

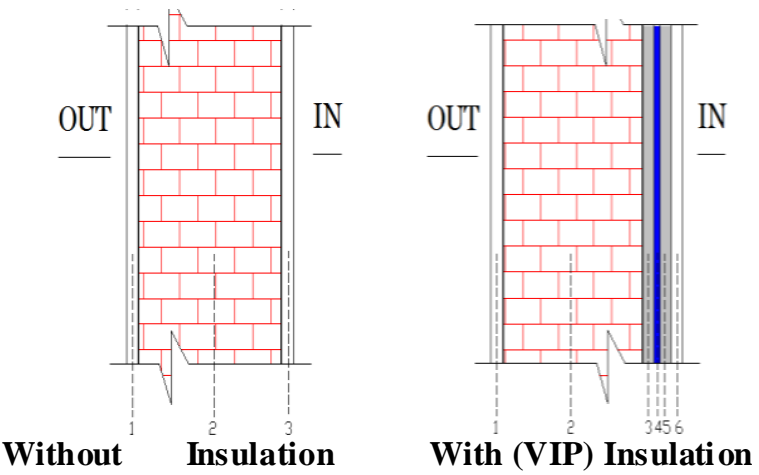

$\underline{\text { Solid Wall Section }(20 \mathrm{~mm}-380 \mathrm{~mm}-20 \mathrm{~mm})}$

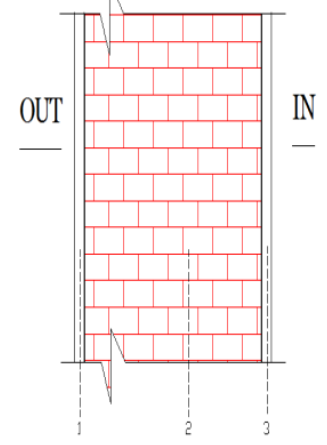

Without Insulation

1- External Plaster

2- Bricks

3- Internal Plaster

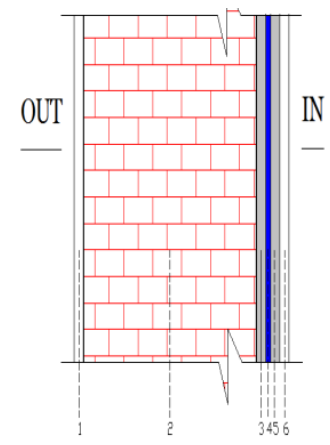

With (VIP) Insulation

1- External Plaster

2- Bricks

3- Protective layer (Expanded Poly sty rene)

4- Vacuum Insulation Panels

5- Protective layer (Expanded

Poly sty rene)

6- Internal Plaster 
Cavity Wall Section with $50 \mathrm{~mm}$ Air Space $\underline{(20 \mathrm{~mm}-120 \mathrm{~mm}-50 \mathrm{~mm}-120 \mathrm{~mm}-20 \mathrm{~mm})}$

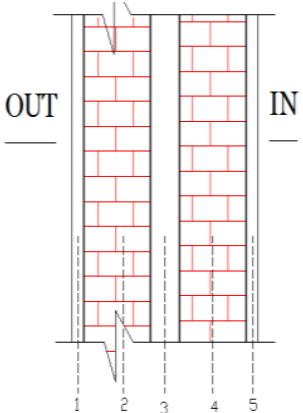

Without Insulation

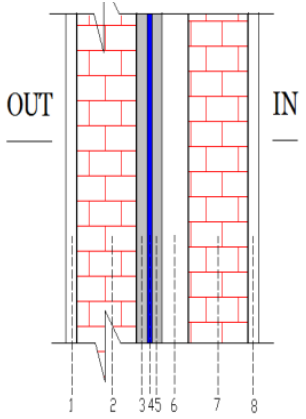

With (VIP) Insulation

Cavity Wall Section with 50mm Air Space $\underline{(20 \mathrm{~mm}-120 \mathrm{~mm}-50 \mathrm{~mm}-250 \mathrm{~mm}-20 \mathrm{~mm})}$

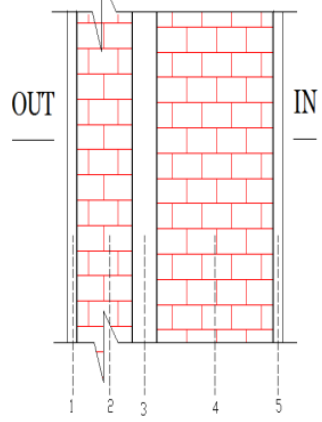

Without Insulation

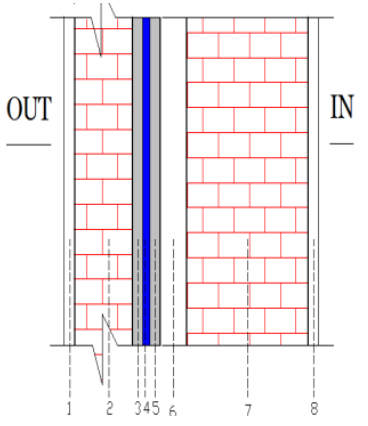

With (VIP) Insulation

Cavity Wall Section with $50 \mathrm{~mm}$ Air Space $(20 \mathrm{~mm}-250 \mathrm{~mm}-50 \mathrm{~mm}-250 \mathrm{~mm}-20 \mathrm{~mm})$

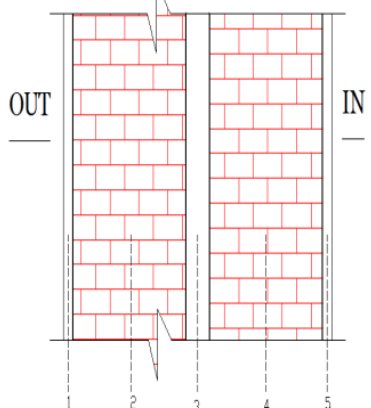

Without Insulation

1- External Plaster

2- Bricks

3- Air Space

4- Bricks

5- Internal Plaster

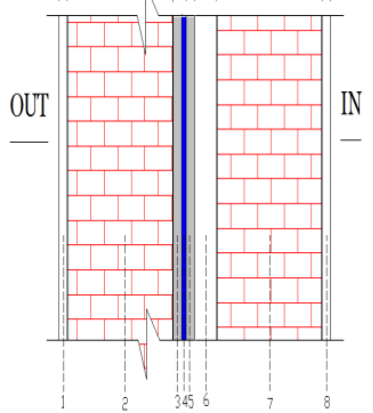

With (VIP) Insulation

1- External Plaster

2- Bricks

3- Protective layer (Expanded Polysty rene)

4- Vacuum Insulation Panels

5- Protective layer (Expanded Polystyrene)6- Air Space

7- Bricks

8- Internal Plaster
3.2. Thermal Properties of wall section as layers

\begin{tabular}{|c|c|c|c|}
\hline \multicolumn{2}{|c|}{ Material } & \multicolumn{2}{|c|}{$\begin{array}{c}\text { Thermal Properties } \\
\text { as layers }\end{array}$} \\
\hline Name & $\begin{array}{l}\text { Thickness } \\
(\mathrm{L})-(\mathrm{mm}) \\
\end{array}$ & $\begin{array}{l}\text { Thermal } \\
\text { Resistance } \\
(\mathrm{R} \text {-value })- \\
\left(\mathrm{m}^{2} . \mathrm{c}^{\circ} / \text { watt }\right) \\
\end{array}$ & $\begin{array}{c}\text { Thermal } \\
\text { Transmittance } \\
\left(\begin{array}{l}\text { (U-value })- \\
\left(\text { watt } / \mathrm{m}^{2} \cdot \mathrm{c}^{\circ}\right)\end{array}\right.\end{array}$ \\
\hline External Plaster & 20 & 0.02 & 50 \\
\hline Internal Plaster & 20 & 0.02 & 50 \\
\hline Air space & 50 & 0.153 & 6.53 \\
\hline \multirow{3}{*}{$\begin{array}{c}\text { Hollow Cement } \\
\text { Bricks }\end{array}$} & 120 & 0.07 & 14.2 \\
\hline & 250 & 0.16 & 6.25 \\
\hline & 380 & 0.24 & 4.16 \\
\hline \multirow{3}{*}{$\begin{array}{l}\text { Protective layer } \\
\text { (Expanded } \\
\text { Polystyrene) }\end{array}$} & 20 & 0.54 & 1.85 \\
\hline & 30 & 0.81 & 1.23 \\
\hline & 50 & 1.35 & 0.74 \\
\hline \multirow{6}{*}{$\begin{array}{l}\text { Vacuum Insulation } \\
\text { Panels (VIP) }\end{array}$} & 10 & 2 & 0.5 \\
\hline & 15 & 3 & 0.33 \\
\hline & 20 & 4 & 0.25 \\
\hline & 25 & 5 & 0.2 \\
\hline & 30 & 6 & 0.16 \\
\hline & 40 & 8 & 0.125 \\
\hline
\end{tabular}

\subsection{Thermal Properties of wall section as whole}

We can calculate (Thermal Resistance (R-Value) Thermal Transmittance (U-Value)) from the two equation:

R-Value $=1 / h_{a o}+\sum R_{i}+1 / h_{a i}=\ldots m^{2} \cdot c \%$ watt Where:

$$
\begin{gathered}
1 / \mathrm{h}_{\mathrm{ao}}+1 / \mathrm{h}_{\mathrm{ai}}=0.178 \quad \mathrm{~m}^{2} \cdot \mathrm{c}^{\circ} / \text { watt } \\
\sum \mathrm{R}_{\mathrm{i}}=\sum \mathrm{R} \text { for each layer } \quad \mathrm{m}^{2} . \mathrm{c}^{\circ} / \text { watt }
\end{gathered}
$$

U-Value $=1 /$ R-Value $=\ldots \ldots \quad$ watt $/ \mathrm{m}^{2} \cdot \mathrm{c}^{\circ}$ Where:

\begin{tabular}{|c|c|c|c|}
\hline \multicolumn{2}{|c|}{ Section } & \multicolumn{2}{|c|}{$\begin{array}{l}\text { Thermal Properties } \\
\text { as whole }\end{array}$} \\
\hline Name & Insulation & $\begin{array}{l}\text { Thermal } \\
\text { Resistance } \\
(\mathrm{R}-\mathrm{v} \text { alue })- \\
\left(\mathrm{m}^{2} . \mathrm{c} / \text { watt }\right) \\
\end{array}$ & $\begin{array}{c}\text { Thermal } \\
\text { Transmittance } \\
\left(\begin{array}{l}\text { (U-value })- \\
\left(\text { watt } / \mathrm{m}^{2} \cdot \mathrm{c}^{\circ}\right)\end{array}\right.\end{array}$ \\
\hline $\begin{array}{l}1-\text { Solid Wall } \\
\text { Section }(20 \mathrm{~mm}- \\
120 \mathrm{~mm}-20 \mathrm{~mm}) \\
\text { with } \quad 10 \mathrm{~mm}\end{array}$ & $\begin{array}{l}\text { Without } \\
\text { VIP }\end{array}$ & 0.28 & 3.57 \\
\hline $\begin{array}{l}\text { Vacuum } \\
\text { Insulation Panels } \\
\text { (VIP) used Hollow } \\
\text { Cement Bricks }\end{array}$ & With VIP & 3.36 & 0.30 \\
\hline $\begin{array}{l}2-\quad \text { Solid Wall } \\
\text { Section }(20 \mathrm{~mm}- \\
120 \mathrm{~mm}-20 \mathrm{~mm}) \\
\text { with } \quad 15 \mathrm{~mm}\end{array}$ & $\begin{array}{l}\text { Without } \\
\text { VIP }\end{array}$ & 0.28 & 3.57 \\
\hline $\begin{array}{l}\text { Vacuum } \\
\text { Insulation Panels } \\
\text { (VIP) used Hollow } \\
\text { Cement Bricks }\end{array}$ & With VIP & 4.36 & 0.23 \\
\hline
\end{tabular}

$$
\text { R-Value }=\ldots \ldots \ldots \ldots . . . \mathrm{m}^{2} . \mathrm{c}^{\circ} / \text { watt }
$$




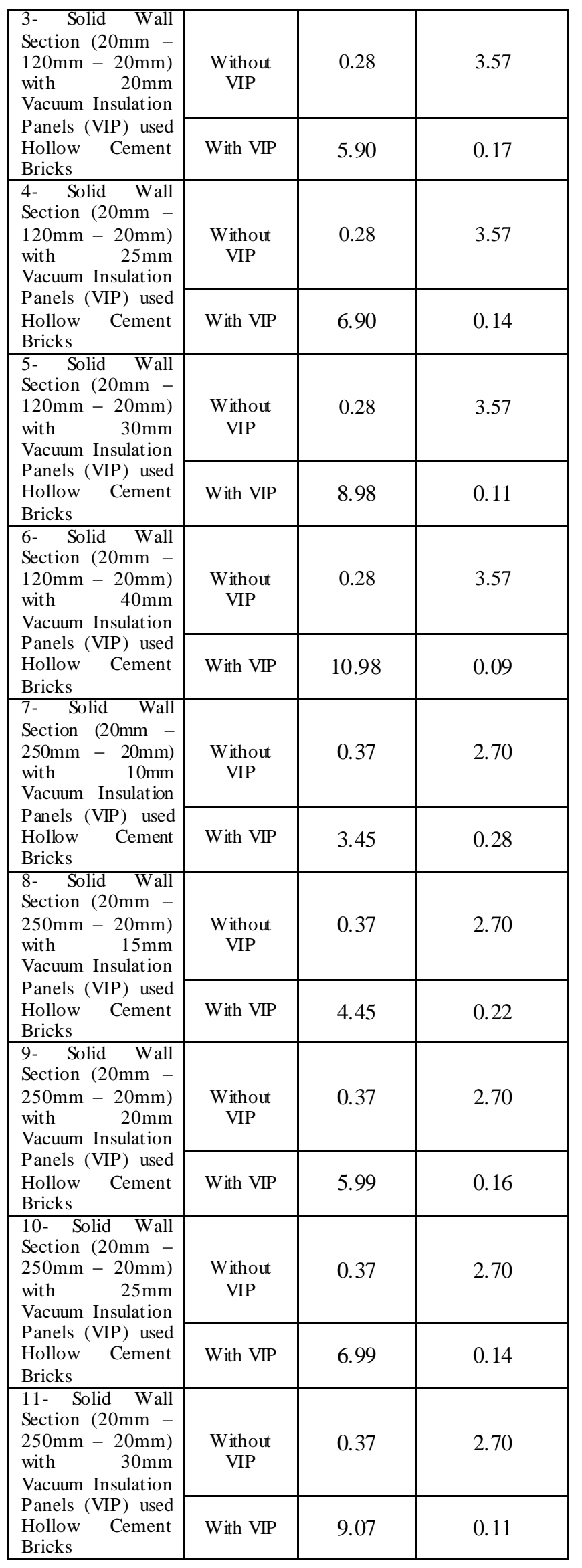

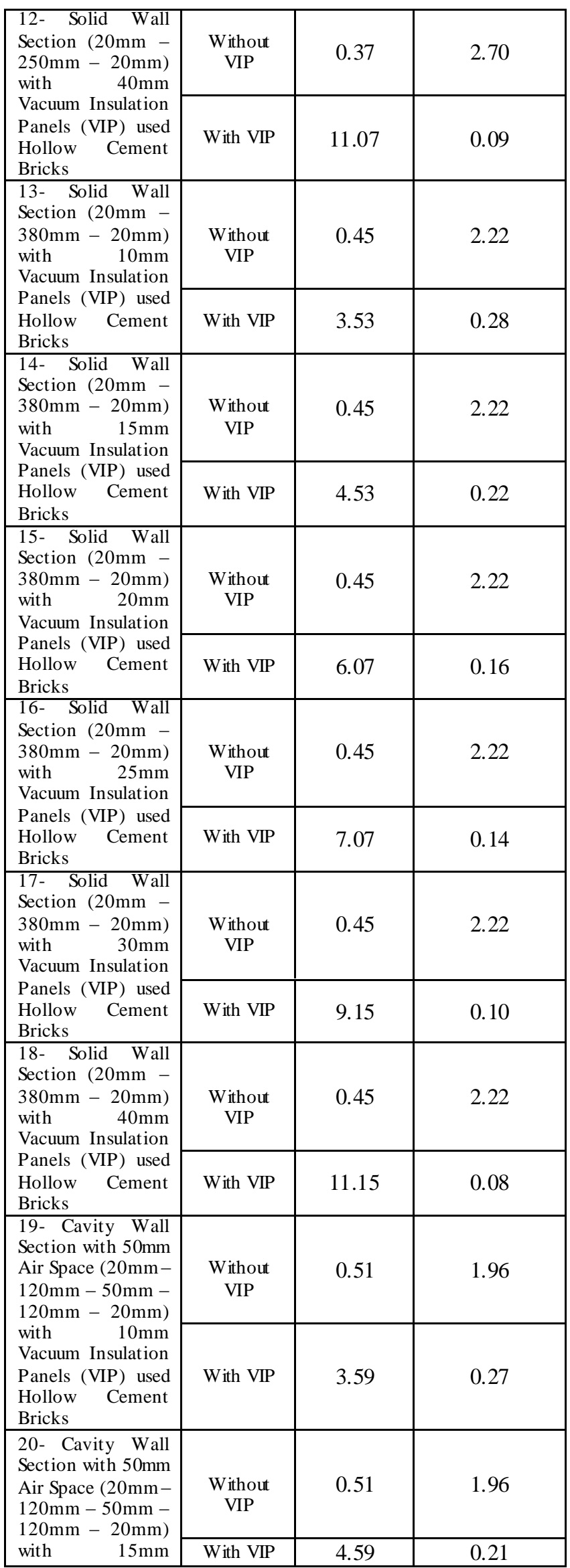




\begin{tabular}{|c|c|c|c|}
\hline $\begin{array}{l}\text { Vacuum Insulation } \\
\text { Panels (VIP) used } \\
\text { Hollow Cement } \\
\text { Bricks }\end{array}$ & & & \\
\hline $\begin{array}{l}21-\text { Cavity Wall } \\
\text { Section with } 50 \mathrm{~mm} \\
\text { Air Space }(20 \mathrm{~mm}- \\
120 \mathrm{~mm}-50 \mathrm{~mm}- \\
120 \mathrm{~mm}-20 \mathrm{~mm})\end{array}$ & $\begin{array}{l}\text { Without } \\
\text { VIP }\end{array}$ & 0.51 & 1.96 \\
\hline $\begin{array}{l}\text { with } 20 \mathrm{~mm} \\
\text { Vacuum Insulation } \\
\text { Panels (VIP) used } \\
\text { Hollow Cement } \\
\text { Bricks }\end{array}$ & With VIP & 6.13 & 0.16 \\
\hline $\begin{array}{l}22-\text { Cavity Wall } \\
\text { Section with } 50 \mathrm{~mm} \\
\text { Air Space }(20 \mathrm{~mm}- \\
120 \mathrm{~mm}-50 \mathrm{~mm}- \\
120 \mathrm{~mm}-20 \mathrm{~mm})\end{array}$ & $\begin{array}{l}\text { Without } \\
\text { VIP }\end{array}$ & 0.51 & 1.96 \\
\hline $\begin{array}{lr}\text { with } & 25 \mathrm{~mm} \\
\text { Vacuum Insulation } \\
\text { Panels (VIP) used } \\
\text { Hollow Cement } \\
\text { Bricks }\end{array}$ & With VIP & 7.13 & 0.14 \\
\hline $\begin{array}{l}23-\text { Cavity Wall } \\
\text { Section with } 50 \mathrm{~mm} \\
\text { Air Space }(20 \mathrm{~mm}- \\
120 \mathrm{~mm}-50 \mathrm{~mm}- \\
120 \mathrm{~mm}-20 \mathrm{~mm})\end{array}$ & $\begin{array}{c}\text { Without } \\
\text { VIP }\end{array}$ & 0.51 & 1.96 \\
\hline $\begin{array}{lr}\text { with } & 30 \mathrm{~mm} \\
\text { Vacuum Insulation } \\
\text { Panels (VIP) used } \\
\text { Hollow Cement } \\
\text { Bricks }\end{array}$ & With VIP & 9.21 & 0.10 \\
\hline $\begin{array}{l}24-\text { Cavity Wall } \\
\text { Section with } 50 \mathrm{~mm} \\
\text { Air Space }(20 \mathrm{~mm}- \\
120 \mathrm{~mm}-50 \mathrm{~mm}- \\
120 \mathrm{~mm}-20 \mathrm{~mm})\end{array}$ & $\begin{array}{c}\text { Without } \\
\text { VIP }\end{array}$ & 0.51 & 1.96 \\
\hline $\begin{array}{l}\text { with } 40 \mathrm{~mm} \\
\text { Vacuum Insulation } \\
\text { Panels (VIP) used } \\
\text { Hollow Cement } \\
\text { Bricks }\end{array}$ & With VIP & 11.21 & 0.08 \\
\hline $\begin{array}{l}25-\text { Cavity Wall } \\
\text { Section with } 50 \mathrm{~mm} \\
\text { Air Space }(20 \mathrm{~mm}- \\
120 \mathrm{~mm}-50 \mathrm{~mm}- \\
250 \mathrm{~mm}-20 \mathrm{~mm})\end{array}$ & $\begin{array}{l}\text { Without } \\
\text { VIP }\end{array}$ & 0.60 & 1.66 \\
\hline $\begin{array}{l}\text { with } 10 \mathrm{~mm} \\
\text { Vacuum Insulation } \\
\text { Panels (VIP) used } \\
\text { Hollow Cement } \\
\text { Bricks }\end{array}$ & With VIP & 3.68 & 0.27 \\
\hline $\begin{array}{l}26-\text { Cavity Wall } \\
\text { Section with } 50 \mathrm{~mm} \\
\text { Air Space }(20 \mathrm{~mm}- \\
120 \mathrm{~mm}-50 \mathrm{~mm}- \\
250 \mathrm{~mm}-20 \mathrm{~mm})\end{array}$ & $\begin{array}{l}\text { Without } \\
\text { VIP }\end{array}$ & 0.60 & 1.66 \\
\hline $\begin{array}{lr}\text { with } & 15 \mathrm{~mm} \\
\text { Vacuum Insulation } \\
\text { Panels (VIP) used } \\
\text { Hollow Cement } \\
\text { Bricks }\end{array}$ & With VIP & 6.22 & 0.16 \\
\hline $\begin{array}{l}27-\text { Cavity Wall } \\
\text { Section with } 50 \mathrm{~mm} \\
\text { Air Space }(20 \mathrm{~mm}- \\
120 \mathrm{~mm}-50 \mathrm{~mm}- \\
250 \mathrm{~mm}-20 \mathrm{~mm})\end{array}$ & $\begin{array}{c}\text { Without } \\
\text { VIP }\end{array}$ & 0.60 & 1.66 \\
\hline $\begin{array}{l}\text { with } 20 \mathrm{~mm} \\
\text { Vacuum Insulation } \\
\text { Panels (VIP) used }\end{array}$ & With VIP & 6.13 & 0.16 \\
\hline
\end{tabular}

\begin{tabular}{|c|c|c|c|}
\hline \multicolumn{4}{|l|}{$\begin{array}{ll}\text { Hollow } & \text { Cement } \\
\text { Bricks } & \end{array}$} \\
\hline $\begin{array}{l}28-\text { Cavity Wall } \\
\text { Section with } 50 \mathrm{~mm} \\
\text { Air Space }(20 \mathrm{~mm}- \\
120 \mathrm{~mm}-50 \mathrm{~mm}- \\
250 \mathrm{~mm}-20 \mathrm{~mm})\end{array}$ & $\begin{array}{l}\text { Without } \\
\text { VIP }\end{array}$ & 0.60 & 1.66 \\
\hline $\begin{array}{l}\text { with } \quad 25 \mathrm{~mm} \\
\text { Vacuum Insulation } \\
\text { Panels (VIP) used } \\
\text { Hollow Cement } \\
\text { Bricks }\end{array}$ & With VIP & 7.13 & 0.14 \\
\hline $\begin{array}{l}29-\text { Cavity Wall } \\
\text { Section with } 50 \mathrm{~mm} \\
\text { Air Space }(20 \mathrm{~mm}- \\
120 \mathrm{~mm}-50 \mathrm{~mm}- \\
250 \mathrm{~mm}-20 \mathrm{~mm}) \\
\text { with } 30 \mathrm{~mm} \\
\text { Vacuum Insulation } \\
\text { Panels (VIP) used } \\
\text { Hollow Cement } \\
\text { Bricks }\end{array}$ & $\begin{array}{l}\text { Without } \\
\text { VIP }\end{array}$ & 0.60 & 1.66 \\
\hline & With VIP & 9.21 & 0.10 \\
\hline $\begin{array}{l}30-\text { Cavity } \text { Wall } \\
\text { Section with } 50 \mathrm{~mm} \\
\text { Air Space }(20 \mathrm{~mm}- \\
120 \mathrm{~mm}-50 \mathrm{~mm}- \\
250 \mathrm{~mm}-20 \mathrm{~mm})\end{array}$ & $\begin{array}{l}\text { Without } \\
\text { VIP }\end{array}$ & 0.60 & 1.66 \\
\hline $\begin{array}{lr}\text { with } & 40 \mathrm{~mm} \\
\text { Vacuum Insulation } \\
\text { Panels (VIP) used } \\
\text { Hollow Cement } \\
\text { Bricks }\end{array}$ & With VIP & 11.21 & 0.08 \\
\hline $\begin{array}{l}31-\text { Cavity Wall } \\
\text { Section with } 50 \mathrm{~mm} \\
\text { Air Space }(20 \mathrm{~mm}- \\
250 \mathrm{~mm}-50 \mathrm{~mm}- \\
250 \mathrm{~mm}-20 \mathrm{~mm})\end{array}$ & $\begin{array}{l}\text { Without } \\
\text { VIP }\end{array}$ & 0.69 & 1.44 \\
\hline $\begin{array}{lr}\text { with } & 10 \mathrm{~mm} \\
\text { Vacuum Insulation } \\
\text { Panels (VIP) used } \\
\text { Hollow Cement } \\
\text { Bricks }\end{array}$ & With VIP & 3.77 & 0.26 \\
\hline $\begin{array}{l}32-\text { Cavity Wall } \\
\text { Section with } 50 \mathrm{~mm} \\
\text { Air Space }(20 \mathrm{~mm}- \\
250 \mathrm{~mm}-50 \mathrm{~mm}- \\
250 \mathrm{~mm}-20 \mathrm{~mm})\end{array}$ & $\begin{array}{l}\text { Without } \\
\text { VIP }\end{array}$ & 0.69 & 1.44 \\
\hline $\begin{array}{l}\text { with } \quad 15 \mathrm{~mm} \\
\text { Vacuum Insulation } \\
\text { Panels (VIP) used } \\
\text { Hollow Cement } \\
\text { Bricks }\end{array}$ & With VIP & 4.77 & 0.20 \\
\hline $\begin{array}{l}33-\text { Cavity Wall } \\
\text { Section with } 50 \mathrm{~mm} \\
\text { Air Space }(20 \mathrm{~mm}- \\
250 \mathrm{~mm}-50 \mathrm{~mm}- \\
250 \mathrm{~mm}-20 \mathrm{~mm})\end{array}$ & $\begin{array}{l}\text { Without } \\
\text { VIP }\end{array}$ & 0.69 & 1.44 \\
\hline $\begin{array}{l}\text { with } 20 \mathrm{~mm} \\
\text { Vacuum Insulation } \\
\text { Panels (VIP) used } \\
\text { Hollow Cement } \\
\text { Bricks }\end{array}$ & With VIP & 6.31 & 0.15 \\
\hline $\begin{array}{l}34-\text { Cavity Wall } \\
\text { Section with } 50 \mathrm{~mm} \\
\text { Air Space }(20 \mathrm{~mm}- \\
250 \mathrm{~mm}-50 \mathrm{~mm}- \\
250 \mathrm{~mm}-20 \mathrm{~mm})\end{array}$ & $\begin{array}{l}\text { Without } \\
\text { VIP }\end{array}$ & 0.69 & 1.44 \\
\hline $\begin{array}{l}\text { with } \quad 25 \mathrm{~mm} \\
\text { Vacuum Insulation } \\
\text { Panels (VIP) used }\end{array}$ & With VIP & 7.31 & 0.13 \\
\hline
\end{tabular}




\begin{tabular}{|c|c|c|c|}
\hline \multicolumn{4}{|l|}{$\begin{array}{l}\text { Hollow Cement } \\
\text { Bricks }\end{array}$} \\
\hline $\begin{array}{l}35-\text { Cavity Wall } \\
\text { Section with } 50 \mathrm{~mm} \\
\text { Air Space }(20 \mathrm{~mm}- \\
250 \mathrm{~mm}-50 \mathrm{~mm}- \\
250 \mathrm{~mm}-20 \mathrm{~mm})\end{array}$ & $\begin{array}{l}\text { Without } \\
\text { VIP }\end{array}$ & 0.69 & 1.44 \\
\hline $\begin{array}{l}\text { with } \quad 30 \mathrm{~mm} \\
\text { Vacuum Insulation } \\
\text { Panels (VIP) used } \\
\text { Hollow Cement } \\
\text { Bricks }\end{array}$ & With VIP & 9.39 & 0.10 \\
\hline $\begin{array}{l}36-\text { Cavity Wall } \\
\text { Section with } 50 \mathrm{~mm} \\
\text { Air Space }(20 \mathrm{~mm}- \\
250 \mathrm{~mm}-50 \mathrm{~mm}- \\
250 \mathrm{~mm}-20 \mathrm{~mm})\end{array}$ & $\begin{array}{l}\text { Without } \\
\text { VIP }\end{array}$ & 0.69 & 1.44 \\
\hline $\begin{array}{l}\text { with } \quad 40 \mathrm{~mm} \\
\text { Vacuum Insulation } \\
\text { Panels (VIP) used } \\
\text { Hollow Cement } \\
\text { Bricks }\end{array}$ & With VIP & 11.39 & 0.08 \\
\hline
\end{tabular}

\section{DISCUSSION}

- Insulation Efficiency of solid wall section $(20 \mathrm{~mm}-$ $120 \mathrm{~mm}-20 \mathrm{~mm}$ ) is average $91.60 \%: \underline{97.48 \%}$

- Insulation Efficiency of solid wall section (20mm $250 \mathrm{~mm}-20 \mathrm{~mm}$ ) is average $\underline{89.63 \%}: \underline{96.67 \%}$

- Insulation Efficiency of solid wall section (20mm $380 \mathrm{~mm}-20 \mathrm{~mm}$ ) is average $\underline{87.39 \%}: \underline{96.40 \%}$

- Insulation Efficiency of cavity wall section with $50 \mathrm{~mm}$ air space $(20 \mathrm{~mm}-120 \mathrm{~mm}-50 \mathrm{~mm}-120 \mathrm{~mm}-20 \mathrm{~mm})$ is average $\underline{86.22 \%}: \underline{95.92 \%}$

- Insulation Efficiency of cavity wall section with $50 \mathrm{~mm}$ air space $(20 \mathrm{~mm}-120 \mathrm{~mm}-50 \mathrm{~mm}-250 \mathrm{~mm}-20 \mathrm{~mm})$

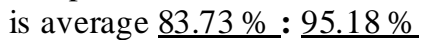

- Insulation Efficiency of cavity wall section with $50 \mathrm{~mm}$ air space $(20 \mathrm{~mm}-250 \mathrm{~mm}-50 \mathrm{~mm}-250 \mathrm{~mm}-20 \mathrm{~mm})$ is average $\underline{81.94 \%}: \underline{94.44 \%}$

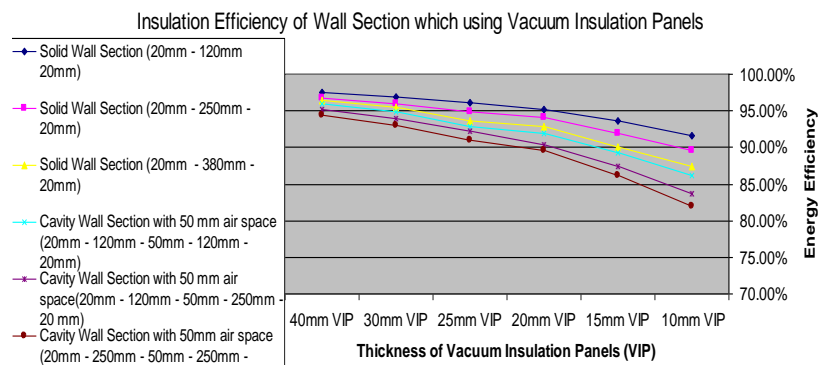

\section{CONCLUSION}

In less than a century, human has led mankind to unexpected heights in human and technical domains. But in the same time, a small part of mankind has:

- Forever consumed some major natural resources.

- Altered the thermal equilib riu m of the planet.

We should now find efficient ways to: Build sustainable development and solve the energy equation for more than 6 billion people.

Energy Efficient Buildings are a part of the solution
Insulation Efficiency can be calculate through equation: Insulation Efficiency $=(\mathrm{U}$-value) Without VIP - (U-value) With VIP $* 100$ (U-value) Without VIP

\begin{tabular}{|c|c|c|c|}
\hline & \multicolumn{2}{|c|}{ Sections } & \multirow{2}{*}{$\begin{array}{c}\text { Insulation } \\
\text { Efficiency } \\
91.60 \%\end{array}$} \\
\hline \multirow{6}{*}{$\begin{array}{l}\text { Solid Wall } \\
\text { Section }(20 \mathrm{~mm} \\
-120 \mathrm{~mm}- \\
20 \mathrm{~mm})\end{array}$} & 1 & with $10 \mathrm{~mm}$ VIP & \\
\hline & 2 & with $15 \mathrm{~mm}$ VIP & $93.56 \%$ \\
\hline & 3 & with $20 \mathrm{~mm}$ VIP & $95.24 \%$ \\
\hline & 4 & with $25 \mathrm{~mm}$ VIP & $96.08 \%$ \\
\hline & 5 & with $30 \mathrm{~mm}$ VIP & $96.92 \%$ \\
\hline & 6 & with 40mm VIP & $97.48 \%$ \\
\hline \multirow{6}{*}{$\begin{array}{c}\text { Solid Wall } \\
\text { Section }(20 \mathrm{~mm} \\
-250 \mathrm{~mm}- \\
20 \mathrm{~mm})\end{array}$} & 7 & with $10 \mathrm{~mm}$ VIP & $89.63 \%$ \\
\hline & 8 & with $15 \mathrm{~mm}$ VIP & $91.85 \%$ \\
\hline & 9 & with $20 \mathrm{~mm}$ VIP & $94.07 \%$ \\
\hline & 10 & with $25 \mathrm{~mm}$ VIP & $94.81 \%$ \\
\hline & 11 & with $30 \mathrm{~mm}$ VIP & $95.93 \%$ \\
\hline & 12 & with 40mm VIP & $96.67 \%$ \\
\hline \multirow{6}{*}{$\begin{array}{c}\text { Solid Wall } \\
\text { Section }(20 \mathrm{~mm} \\
-380 \mathrm{~mm}- \\
20 \mathrm{~mm})\end{array}$} & 13 & with $10 \mathrm{~mm}$ VIP & $87.39 \%$ \\
\hline & 14 & with $15 \mathrm{~mm}$ VIP & $90.09 \%$ \\
\hline & 15 & with $20 \mathrm{~mm}$ VIP & $92.79 \%$ \\
\hline & 16 & with $25 \mathrm{~mm}$ VIP & $93.69 \%$ \\
\hline & 17 & with $30 \mathrm{~mm}$ VIP & $95.50 \%$ \\
\hline & 18 & with 40mm VIP & $96.40 \%$ \\
\hline \multirow{6}{*}{$\begin{array}{c}\text { Cavity Wall } \\
\text { Section with } \\
50 \mathrm{~mm} \text { Air } \\
\text { Space }(20 \mathrm{~mm}- \\
120 \mathrm{~mm}- \\
50 \mathrm{~mm}- \\
120 \mathrm{~mm}- \\
20 \mathrm{~mm})\end{array}$} & 19 & with $10 \mathrm{~mm}$ VIP & $86.22 \%$ \\
\hline & 20 & with $15 \mathrm{~mm}$ VIP & $89.29 \%$ \\
\hline & 21 & with $20 \mathrm{~mm}$ VIP & $91.84 \%$ \\
\hline & 22 & with $25 \mathrm{~mm}$ VIP & $92.86 \%$ \\
\hline & 23 & with $30 \mathrm{~mm}$ VIP & $94.90 \%$ \\
\hline & 24 & with $40 \mathrm{~mm}$ VIP & $95.92 \%$ \\
\hline \multirow{6}{*}{$\begin{array}{l}\text { Cavity Wall } \\
\text { Section with } \\
50 \mathrm{~mm} \text { Air } \\
\text { Space }(20 \mathrm{~mm}- \\
120 \mathrm{~mm}- \\
50 \mathrm{~mm}- \\
250 \mathrm{~mm}- \\
20 \mathrm{~mm})\end{array}$} & 25 & with $10 \mathrm{~mm}$ VIP & $83.73 \%$ \\
\hline & 26 & with $15 \mathrm{~mm}$ VIP & $87.35 \%$ \\
\hline & 27 & with $20 \mathrm{~mm}$ VIP & $90.36 \%$ \\
\hline & 28 & with $25 \mathrm{~mm}$ VIP & $92.17 \%$ \\
\hline & 29 & with $30 \mathrm{~mm}$ VIP & $93.98 \%$ \\
\hline & 30 & with $40 \mathrm{~mm}$ VIP & $95.18 \%$ \\
\hline Cavity Wall & 31 & with $10 \mathrm{~mm}$ VIP & $81.94 \%$ \\
\hline Section with & 32 & with $15 \mathrm{~mm}$ VIP & $86.11 \%$ \\
\hline 50mm Air & 33 & with $20 \mathrm{~mm}$ VIP & $89.58 \%$ \\
\hline Space $(20 \mathrm{~mm}-$ & 34 & with $25 \mathrm{~mm}$ VIP & $90.97 \%$ \\
\hline $\begin{array}{c}250 \mathrm{~mm}- \\
50 \mathrm{~mm}-\end{array}$ & 35 & with $30 \mathrm{~mm}$ VIP & $93.06 \%$ \\
\hline $250 \mathrm{~mm}-$ & 36 & with 40mm VIP & $94.44 \%$ \\
\hline
\end{tabular}

\section{REFERENCES}

[1] Ahmed Sobhy Foda, "Energy Code and Building Envelope", Master of Science in Architecture, Department of Architecture, Faculty of Engineering, University of Cairo, Giza, 2005.

[2] Anne Grete Hestnes and Marit Thyholt, "Zero Emission Buildings (ZEB)", The Research Centre on Zero Emission Buildings, February 2009.

[3] Phalguni Mukhopadhyaya, "High-performance insulation materials", National Research, Council Canada, Septe mber 2004.

[4] Bjørn Petter Jelleab and Arild Gustavsenc, "Advanced Thermal Building Insulation (From Vacuum Insulation Panels to Nano Insulation Materials)", SFFE 
Lunch Lecture, Trondheim, Norway, 9th of September, 2010.

[5] Phalguni Mukhopadhyaya, "High-Performance Vacuum Insulation Panel in Building Envelope Construction", National Research, Council Canada, 2010 AMTS Technical Committee Meeting, 06 October 2010. [6] Prof. Armin Binz, Gregor Stein ke, "Applications of Vacuum Insulation in the Building Sector", 7th International Vacuum Insulation Symposium, Empa,
Duebendorf / Zurich, Switzerland, September 28-29, 2005.

[7] H. Wein lader, H.-P. Ebert, J. Fricke, " VIG - Vacuum Insulation Glass", Bavarian Center for Applied Energy Research (ZAE Bayern), Am Hubland, D-97074 Wurzburg.

[8-9] Rasha Mohamed Sleem, Impact of Building Technology used in Building Envelope on Energy Reduction in Buildings, Master of Science in Architecture, Department of Architecture, Faculty of Engineering, University of Cairo, Giza, 2003 